\title{
Distinctive personality profiles of fibromyalgia and chronic fatigue syndrome patients
}

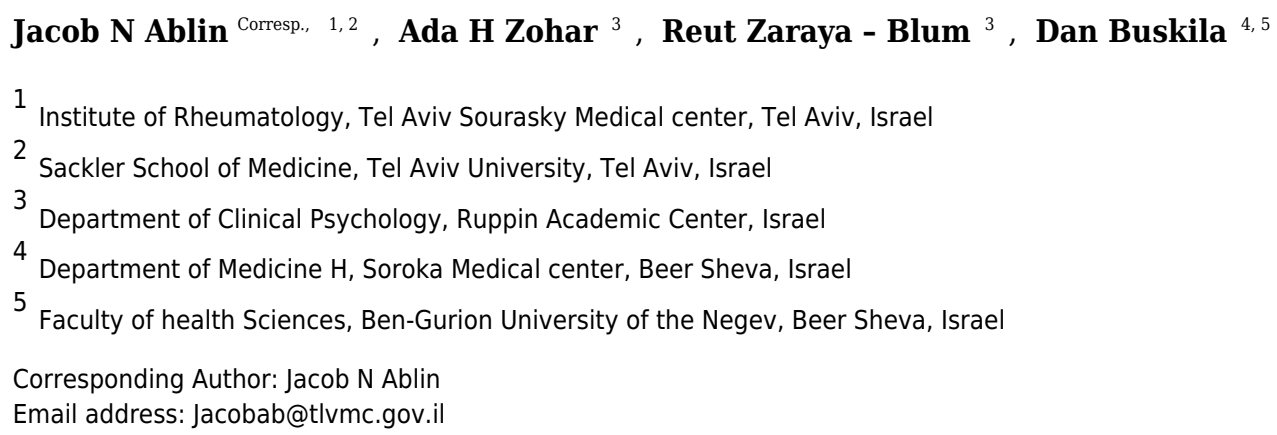

Objective: The current study is an innovative exploratory investigation, aiming at identifying differences in personality profiles within FMS and CFS patients. Method: 344 participants (309 female, 35 male) reported suffering from FMS and/or CFS and consented to participate in the study. Participants were recruited at an Israeli FM/CFS patient meeting held in May 2013, and through an announcement posted on several social networks.

Participants were asked to complete a research questionnaire, which included FMS criteria and severity scales, and measures of personality, emotional functioning, positivity, social support and subjective assessment of general health. 204 participants completed the research questionnaire ( $40.7 \%$ attrition rate). Results: A cluster analysis produced two distinct clusters, which differed significantly on psychological variables, but did not differ on demographic variables or illness severity. As compared to cluster number $2(\mathrm{~N}=107)$, participants classified into cluster number $1(\mathrm{~N}=97)$ showed a less adaptive pattern, with higher levels of Harm Avoidance and Alexithymia; higher prevalence of Type D personality; and lower levels of Persistence, Reward Dependence, Cooperation, Self-Directedness, social support and positivity. Conclusion: The significant pattern of results indicates at least two distinct personality profiles of FM and CFS patients. Findings from this research may help improve the evaluation and treatment of FM and CFS patients, based on each patient's unique needs, psychological resources and weaknesses, as proposed by the current trend of personalized medicine. 
1 Distinctive Personality Profiles of Fibromyalgia and Chronic Fatigue Syndrome Patients

2 Jacob N. Ablin ${ }^{1}$, Ada H. Zohar², Reut Zaraya - Blum² ${ }^{2}$ Dan Buskila ${ }^{3}$

3 1- Institute of Rheumatology, Tel Aviv Sourasky Medical center \& Sackler School of

$4 \quad$ Medicine, Tel Aviv University, Israel

5 2- Department of Clinical Psychology, Ruppin Academic Center, Israel

6 3- Department of Medicine H, Soroka Medical center and Faculty of health Sciences, Ben

$7 \quad$ Gurion University of the Negev, Beer Sheva, Israel

8 Corresponding author:

$9 \quad$ Jacob N. Ablin MD

10 Tel Aviv Sourasky medical center

116 Weizmann St. Tel Aviv 64239, Israel

$12 \quad$ Tel $-972-3-6973668$

$13 \quad$ Fax $-972-3-6974577$

14 e-mail - Jacobab@tlvmc.gov.il 
15 Abstract

16 Objective: The current study is an innovative exploratory investigation, aiming at identifying

17 differences in personality profiles within Fibromyalgia Syndrome (FMS) and Chronic Fatigue

18 Syndrome (CFS) patients.

19 Method: 344 participants (309 female, 35 male) reported suffering from FMS and/or CFS and

20 consented to participate in the study. Participants were recruited at an Israeli FM/CFS patient

21 meeting held in May 2013, and through an announcement posted on several social networks.

22 Participants were asked to complete a research questionnaire, which included FMS criteria and

23 severity scales, and measures of personality, emotional functioning, positivity, social support and

24 subjective assessment of general health. 204 participants completed the research questionnaire

25 (40.7\% attrition rate).

26 Results: A cluster analysis produced two distinct clusters, which differed significantly on

27 psychological variables, but did not differ on demographic variables or illness severity. As

28 compared to cluster number $2(\mathrm{~N}=107)$, participants classified into cluster number 1 ( $\mathrm{N}=97)$

29 showed a less adaptive pattern, with higher levels of Harm Avoidance and Alexithymia; higher

30 prevalence of Type D personality; and lower levels of Persistence, Reward Dependence,

31 Cooperation, Self-Directedness, social support and positivity.

32 Conclusion: The significant pattern of results indicates at least two distinct personality profiles of

33 FM and CFS patients. Findings from this research may help improve the evaluation and treatment

34 of FM and CFS patients, based on each patient's unique needs, psychological resources and

35 weaknesses, as proposed by the current trend of personalized medicine. 


\section{Introduction}

37 Fibromyalgia (FMS) is a syndrome characterized by chronic widespread musculoskeletal pain

38 and tenderness, associated with disturbed sleep patterns, chronic fatigue and a spectrum of

39 additional functional symptoms. While the pathogenesis and etiology of FMS remain

40 incompletely understood, a leading paradigm in this aspect currently holds that FMS is the result

41 of a process of pain centralization, whereby the central nervous system has become extremely

42 hyper-sensitive to the processing and transmission of pain, thus causing amplification of painful

43 as well as non-painful stimuli and leading to a state of chronic pain (Clauw 2015). Chronic

44 Fatigue Syndrome (CFS) is a clinical syndrome characterized by fatigue lasting over six months

45 which is not associated with physical effort and is not relieved by rest (Afari \& Buchwald 2003).

46 Considerable clinical overlap existed between FMS and CFS, with many patients fulfilling

47 criteria for both conditions simultaneously. Similar to other chronic medical conditions,

48 psychiatric comorbidity such as anxiety and depression have been documented in a portion of

49 FMS patients, while other patients appear to be surprisingly psychologically resilient in the face

50 of ongoing pain and fatigue (Giesecke et al. 2003).

51 The purpose of the current study was to evaluate personality types found among FMS/CFS

52 patients. The study was not specifically designed to compare FMS patients to CFS patients, but

53 rather to characterize this generally overlapping population of patients in terms of personality

54 types.

55 In the current study, we have focused on the following four aspects of personality and

56 psychological coping: Alexithymia - the inability to identify and describe emotions in the self;

57 Type D personality- the tendency towards negative affectivity and social inhibition and

58 personality components, based on the psycho-biological model of Cloninger regarding 
59 temperament and character; Level of positivity - self-confidence, optimism and satisfaction with

60 life; Social support.

61 Alexithymia has been studied in patients suffering from chronic pain (Celikel \& Saatcioglu 2006;

62 Lumley et al. 2002) and FMS (Sayar et al. 2004; Steinweg et al. 2011). In a recent study, Castelli

63 et al. reported alexithymia traits in 20\% of a sample of FMS patients (Castelli et al. 2012). Thus,

64 it has previously been suggested that the inability to correctly identify physical manifestations of

65 emotions makes alexithymic individuals susceptible to incorrectly attributing innocent physical

66 symptoms to physical disease (Tuzer et al. 2011). Originally identified by Denollet et al. as a

67 predictor of long-term mortality among patients suffering from coronary heart disease (Denollet

68 et al. 1996), type D personality was characterized by a tendency towards negative affectivity

69 (NA) together with social inhibition (SI). Type D personality is strongly associated with both

70 musculoskeletal pain, psychosomatic symptoms (Condén et al. 2013b) and sleep disorders

71 (Condén et al. 2013a).

72 The psycho-biological model of temperament and character (Cloninger et al. 1993) suggests the

73 existence of characteristic temperament dimensions, defined in terms of individual differences in

74 associative learning in response to novelty, danger or punishment and reward. Thus, Novelty

75 Seeking (NS) is described as a bias towards behaviors such as exploratory activity in response to

76 novelty, impulsive decision making, as well as quick loss of temper in response to frustration.

77 Harm Avoidance (HA) biases individuals to habitual pessimistic worry, fear of uncertainty,

78 shyness and rapid fatigability. Reward Dependence (RD) is a tendency to sentimentality, to social

79 attachment and dependence on the approval of others. Persistence (PS), refers to a tendency to

80 continue specific behavior despite feelings of frustration, fatigue or lack of reward (Cloninger \&

81 Svrakic 1997). According to the "unified biosocial theory of personality", NS is associated with

82 low basal dopaminergic activity, HA with high serotonergic activity, and RD with low basal 
83 noradrenergic activity (Cloninger 1986). The psycho-biological model also identifies three

84 dimensions of character, namely self-directedness (SD), Cooperativeness (CO) and Self-

85 Transcendence (ST). Genetic analysis has confirmed a hereditability component between 50\%

86 and 65\% in each of the personality traits (Heath et al. 1994). Cohen et al. investigated the

87 association between FMS and the serotonin transporter promoter region polymorphism, and the

88 relationship to anxiety- related personality traits in FMS patients (Cohen et al. 2002). A

89 subsequent study investigated the association between FMS and the dopamine D4 receptor gene

90 and the relationship to NS trait (Dan et al. 2004). Glazer et al. comparing the personality of FMS

91 patients and their relatives with and without FMS (Glazer et al. 2010) found that FMS patients, as

92 well as their relatives with FMS, had higher scores on HA than relatives without FMS. Despite

93 these studies, a recent systematic review failed to identify a specific "FMS personality"; instead

94 it was proposed that personality may act as a filter, that modulates a person's response to

95 psychological stressors and that certain personalities may facilitate translation of these stressors

96 to physiological responses which culminate in FMS (Malin \& Littlejohn 2012). While various

97 aspects of both personality as well as psychiatric comorbidity have been frequently studied in the

98 FMS syndrome, less attention has been focused on aspects of resilience and positivity, which is

99 defined as a general dispositional determinant of subjective wellbeing, which may account for

100 individual variation and stability in happiness, despite environmental challenge (Kozma et al.

$1012000)$.

102 Social support, the perception of the individual that he is cared for and loved, esteemed, and a

103 member of a network of mutual obligations (Cobb 1976) may have a protective effect versus a

104 broad range of pathological conditions. Social support is directly associated with the severity of 105 physiological and psychological symptoms and may moderate the health-related effects of stress 
106 (Procidano \& Heller 1983; Sarason et al. 1985). Patients suffering from FMS and CFS have

107 previously been reported to suffer from low levels of social support (Schoofs et al. 2004).

108 Methods:

109 Participants: Individuals who self-identified as suffering from FMS or CFS, female or male were

110 recruited through an appeal made at an FMS patient organization meeting held in Tel Aviv, Israel

111 on May 2013, as well as through internet forums of FMS/CFS patients. No reward was offered,

112 for participation. Participants were offered to be notified of the study results. The study was

113 approved by the IRB of the Ruppin Academic Center (IRB reference number 8/2013).

114 Procedure:

115 After providing consent to participate in the study, participants were given access to an on-line

116 self-report, using Qualtrics as the delivery system at http://www.qualtrics.com website (Provo,

117 UT, USA) (Bryson et al. 2012; Passmore et al. 2002). The online survey, which was anonymous,

118 took about 20 minutes to complete, and participants were able to stop and restart as necessary, in

119 order to minimize the discomfort FMS patients may experience during prolonged sitting. In total,

120204 participants completed the entire survey while 140 (40.7\%) completed it only partially.

121 Tools and measures: FMS: Meeting FMS diagnostic criteria as well as disease severity were

122 determined through the Fibromyalgia Criteria and Severity Scales (FCSS) which are used for the

123 diagnosis of FMS in epidemiological and clinical studies (Wolfe et al. 2011) and which are based

124 on the 2010 proposed ACR criteria for the diagnosis of FMS (Wolfe et al. 2010). This

125 questionnaire includes two scales: the widespread pain index (WPI) and the symptom severity

126 scale (SSS). The results of these two scales are used both for establishing the diagnosis of FMS

127 as well as for evaluating severity. Notably, in the current study a diagnosis of CFS was not

128 specifically tested for separately; rather patients were surveyed for the fulfillment of FMS criteria 
129 alone as described. We adopted this approach due to the overwhelming clinical epidemiological 130 overlap between FMS and CFS.

131 Alexithymia was assessed through the Toronto Alexithymia Scale (Bagby et al. 1994) which 132 measures inability to identify and describe emotions. The questionnaires includes 20 items on a 133 Likert scale from 1 to 5 and measures 3 subscales: the Difficulty Describing Feelings subscale, 134 the Difficulty Identifying Feeling subscale and the Externally-Oriented Thinking subscale. This 135 tool has previously been translated into Hebrew and validated (Zohar et al. 2011).

136 Personality evaluation:

137 Personality was evaluated through the following questionnaires: Temperament and Character 138 Inventory Revised TCI-R (Cloninger et al. 1994). In the current study the shortened, 140 item 139 version was used (TCI-140). This questionnaire includes 7 scales: Harm avoidance (HA), Reward 140 Dependence (RD), Persistence (PS), Self-Directedness (SD), Cooperativeness (CO) and Self

141 Transcendence (ST). A validated Hebrew version was used in the current study (Zohar \&

142 Cloninger 2011). Type D personality was assessed using the DS-14 questionnaire (Denollet 2005)

143 which includes 7 items referring to negative affectivity (NA) and 7 items referring to social

144 inhibition (SI). Individuals who score 10 points or more on both NA and SI dimensions are

145 classified as Type D personality. A validated Hebrew translation of this tool was utilized in the 146 current study [31].

147 Positivity: this construct was assessed by the Positivity Orientation Scale (P scale) [58]. This tool 148 is comprised of 8 items assessing an individual's positive attitude about himself about his life and 149 his attitude towards the future.

150 Results: 
151344 participants (309 females and 35 males) participated.

152 Table 1 presents demographic details of the study participants.

153 Table 1: Demographic data, ethnicity, level of religiosity, employment status and level of physical

154 activity among study participants.

\begin{tabular}{|c|c|c|c|}
\hline Age & $\begin{array}{l}16-69 \text { (mean }-41.74, \text { SD - } \\
12.09\end{array}$ & Frequency & $\%$ \\
\hline \multirow[t]{2}{*}{ Gender } & Female & 309 & 89.8 \\
\hline & Male & 35 & 10.2 \\
\hline \multirow[t]{4}{*}{ Marital status } & Unmarried & 96 & 27.9 \\
\hline & Married & 174 & 50.6 \\
\hline & Divorced & 68 & 19.8 \\
\hline & Widow & 6 & 1.7 \\
\hline \multirow[t]{5}{*}{ Educational level } & Partial high school ( $\leq 10$ years) & 18 & 5.2 \\
\hline & Full high school (12 years) & 102 & 29.7 \\
\hline & $\geq 13$ years & 84 & 24.4 \\
\hline & First degree & 94 & 27.3 \\
\hline & Second or third degree & 46 & 13.4 \\
\hline \multirow[t]{4}{*}{ Ethnicity } & Jewish & 330 & 95.9 \\
\hline & Arab - Muslim & 2 & 0.6 \\
\hline & Arab - Christian & 1 & 0.3 \\
\hline & Other & 11 & 3.2 \\
\hline \multirow[t]{4}{*}{ Religiosity level } & Secular & 238 & 69.2 \\
\hline & Traditional & 61 & 17.7 \\
\hline & Orthodox & 38 & 11.1 \\
\hline & "Haredi" (ultra- orthodox) & 6 & 1.7 \\
\hline \multirow[t]{4}{*}{ Employment status } & Not working or studying & 115 & 33.4 \\
\hline & Fully employed & 34 & 9.9 \\
\hline & Partial employment & 100 & 29.1 \\
\hline & Occasional work/ volunteer & 95 & 27.6 \\
\hline \multirow{5}{*}{$\begin{array}{l}\text { Level of physical } \\
\text { activity }\end{array}$} & No physical activity & 51 & 14.8 \\
\hline & Low physical activity & 151 & 43.9 \\
\hline & Moderate activity & 114 & 33.1 \\
\hline & High activity & 27 & 7.8 \\
\hline & Very High activity & 1 & 0.3 \\
\hline
\end{tabular}

155 Patient characteristics:

156260 participants, which represented $75.6 \%$ of the total, met diagnostic criteria for FMS, while 84

157 participants (24.4\%) did not. Notably, participants not meeting FMS criteria nonetheless suffered

158 from chronic widespread pain, fatigue and other symptoms of variable severity and thus may be

159 considered as representing sub- threshold FMS cases. 235 (90.4\%) of the participants who met

160 FMS criteria were female. 99 participants (28.8\%) were characterized as type D personality, 
161 including $29.1 \%$ of females and $25.7 \%$ of males respectively. No significant association was

162 observed between gender and type $\mathrm{D}$ personality $-\chi^{2}(1)=0.179, \mathrm{p}=0.673$ (NS) $\mathrm{N}=344$. $30 \%$ of

163 participants meeting FMS criteria were characterized as type D personality compared with 25\%

164 of participants not fulfilling FMS criteria. No significant association was observed between type

$165 \mathrm{D}$ and fulfilling FMS criteria $\chi^{2}(1)=0.774, \mathrm{p}=0.379$ (NS), $\mathrm{N}=344$.

166 Cluster analysis:

167 In order to evaluate patterns of psychological coping, cluster analysis was performed on 204

168 participants, whose questionnaires were complete. The analysis yielded 2 groups, which differed

169 from each other on psychological variables including type D personality, alexithymia, positivity,

170 social support, Cloninger's temperament and character domains. 97 and 107 participants were

171 classified into cluster 1 and cluster 2 respectively. Compared with cluster 2, cluster 1 was

172 characterized by the following character dimensions: lower cooperativeness (CO) and lower self-

173 directedness (SD). Cluster 1 was also characterized by the following temperament domains:

174 lower persistence (PS), lower reward dependence (RD), and higher harm avoidance (HA). This

175 cluster was also characterized by higher levels of alexithymia, less social support, lower levels of

176 positivity and higher frequency of type $\mathrm{D}$ personality. Table 2 presents mean values of 177 psychological variables for each cluster. 
178 Table 2: Cluster analysis presenting mean values of psychological variables per cluster group.

\begin{tabular}{|l|c|c|}
\hline & Cluster 1(N=98) & Cluster 2 (N=106) \\
& Mean(SD) & Mean(SD) \\
\hline Self-transcendence (ST) & $44.73(12.02)$ & $44.49(12.65)$ \\
\hline Cooperativeness (CO) & $74.46(8.36)$ & $82.60(7.27)$ \\
\hline Self-directedness (SD) & $57.59(9.09)$ & $73.79(7.54)$ \\
\hline Persistence (PS) & $58.81(10.67)$ & $71.22(8.38)$ \\
\hline Reward dependence (RD) & $65.53(9.99)$ & $59.73(11.07)$ \\
\hline Harm avoidance (HA) & $73.76(9.69)$ & $54.23(8.14)$ \\
\hline Novelty seeking (NS) & $55.87(7.97)$ & $34.75(7.19)$ \\
\hline Alexithymia & $46.07(10.93)$ & $59.00(9.21)$ \\
\hline Social Support & $43.65(11.52)$ & $3.62(0.51)$ \\
\hline Positivity & $2.79(0.65)$ & $0.20(0.04)$ \\
\hline Type D personality & $0.755(0.43)$ & \\
\hline
\end{tabular}

179 Demographic characteristics of clusters:

180 After the process of cluster analysis, demographic parameters were compared between the two

181 clusters including age, gender, educational level and employment status. No significant

182 differences were found between cluster 1 and cluster 2 regarding age, gender, marital status,

183 educational level, and employment status. In order to test the hypothesis that the two clusters

184 would not differ regarding FMS criteria, a Chi-Square Test for Independence was performed.

$18579.4 \%$ of individuals in cluster 1 and 72\% of individuals in cluster 2 fulfilled FMS diagnostic

186 criteria. In accordance with the hypothesis, no significant association was found between cluster

187 designation and the diagnosis of FMS $-\chi 2(1)=1.513, \mathrm{p}=0.216(\mathrm{NS}), \mathrm{N}=204$. 
188 In order to compare the cluster analysis categories regarding the clinical parameters of FMS

189 severity, as well as levels of physical activity, a Multivariate analysis of variance (MANOVA)

190 was performed. In this analysis the independent variable was the cluster category and the

191 dependent categories were the subjective health assessment, the extent of physical activity, the

192 symptom severity score (SSS), and the widespread pain index (WPI). The results of the

193 MANOVA indicated a significant difference between the cluster categories regarding the

194 aggregate of dependent variables [Wilks' Lambda=0.93, F $(4,199)=3.56, p<0.01]$. Univariate

195 analysis demonstrated a significant difference between the cluster categories regarding the SSS -

$196 \mathrm{p}<0.0001$, with symptom severity significantly higher among individuals in cluster 1

197 (Mean =9.11, SD=2.18) compared with cluster 2 (Mean=7.99, SD=2.19). No significant

198 difference was found when comparing cluster 1 with cluster 2 regarding subjective health

199 assessment, WPI and levels of physical activity. 
Discussion:

201 The current study constitutes a novel exploratory approach towards identifying psychological

202 patterns and personality aspects of resilience among patients suffering from FMS and CFS. In

203 this study we have attempted to elucidate the ways in which personality patterns interact with

204 styles of coping with illness. While we recruited patients who self - reported a diagnosis of FMS,

205 a significant proportion of the participants were found to be sub-threshold regarding the diagnosis

206 of FMS, according to current diagnostic criteria. In the analysis of results we have chosen to

207 compare this group of individuals, who suffer from varying degrees of chronic pain and fatigue,

208 with individuals who fulfil the FMS diagnostic criteria. Individuals fulfilling the diagnostic

209 criteria were found to rate significantly lower on scales of social support, positivity, physical

210 activity, and subjective health assessment. These groups also differed in the level of self-

211 directedness (SD), which was lower among the criteria-positive individuals compared with the

212 sub-threshold individuals. This finding is in accordance with previous research, which indicates

213 that the SD of FMS patients is lower than that found among healthy controls (Gencay-Can \& Can

214 2012). SD is a trait which indicates the extent to which an individual can depend on himself and

215 on his capabilities, allowing him to feel responsible for his own fate, resourceful and hopeful

216 (Cloninger et al. 1997). Low levels of SD (as well as low levels of cooperativeness and high

217 levels of harm avoidance) have been described in chronic pain patients (Conrad et al. 2007).

218 The most significant findings of the current study are related to the classification of the study

219 participants (both FMS-criteria positive and sub-threshold individuals) into two clusters, based on

220 the psychological styles of coping identified. This analysis yielded two clusters which clearly

221 differ in their psychological profile, while not being significantly different on clinical grounds,

222 and the clusters were nearly equal in the number of individuals they aggregated. The numerically

223 larger cluster (107 vs. 97) was highly resilient in their psychological profile. Individuals in this

224 cluster had a frequency of type D personality which was similar to the general population; 
225 They were as high as healthy controls in the character traits of SD and CO, and in the

226 temperament trait of PS, and as low in the temperament trait of HA. (Cloninger \& Zohar 2011)

227 This result is very similar to that of Leombruni et al. (Leombruni et al. 2016) who also found two

228 distinct personality clusters for FM patients, with the bigger cluster characterized by resilient

229 personality profile and emotional style. The first group of patients was the mal-adapted cluster,

230 characterized by higher levels of HA and alexithymia, higher frequency of type D, and lower

231 levels of CO, SD, PS, RD positivity and social support. No significant differences were identified

232 between the groups regarding the traits of self-transcendence (ST) and novelty seeking (NS). The

233 clear clustering of patients between these two groups and the striking differences found between

234 them, indicate the existence of two uniquely separate styles of coping among FMS patients and

235 sheds doubt on the findings of previous studies which implied the existence of one homogeneous

236 personality pattern among FMS patients (Anderberg et al. 1999; Gencay-Can \& Can 2012) and

237 other patients suffering from chronic pain (Conrad et al. 2013).The emotional profile which

238 emerges from the characteristics of the first group indicates a generally less adaptive pattern,

239 associated with a decreased well-being. Due to high levels of HA, these individuals are more

240 prone to be cautious, nervous, passive, negativistic, insecure and pessimistic. High levels of

241 carefulness and pessimism may be associated with difficulty with expressing emotions, due to

242 fear of negative implications (Cloninger \& Zohar 2011). Individuals with high levels of

243 alexithymia, as in this group, experience difficulty in identifying their own emotions and in

244 differentiating between these emotions and physical sensations which are associated with

245 emotional stimulation. They may have difficulty articulating emotions and tend to be self-

246 centered, inpatient and critical. The low levels of SD of such individuals is associated with low

247 self-esteem, difficulty in taking responsibility, difficulty in setting long-term goals and in

248 overcoming obstacles. Individuals in the first group are also characterized by low levels of PS

249 and difficulty in coping with frustration. Low reward - dependence among these individuals has 
250 the advantage of less dependence on satisfying others and more independence; it also however

251 carries the disadvantages of a tendency towards social withdrawal and isolation (Cloninger 2008).

252 Thus, individuals in this group are prone towards low levels of social support, and may not feel

253 they have adequate bonds to fall back on (Zimet et al. 1988). Such individuals tend to have lower

254 levels of positivity, lower self-esteem and higher degrees of pessimism (Caprara et al. 2012).

255 Individuals in the first group were also found to have a higher frequency of type D personality,

256 the characteristics of which appear to be in agreement with other personality characteristics

257 identified among individuals in this group. Due to negative cognitive patterns, these individuals

258 tend to experience difficulty in acquiring social support, tend to experience anxiety in social

259 contexts and experience the surroundings as critical, thus augmenting negative feelings (Denollet

260 2005). Individuals in the second group, show a surprising psychological profile, of resilience and

261 well-being, although they are no less prone to FM pain and fatigue. They are characterized by a

262 healthier and more adaptive pattern of coping, compared with the first group. Due to lower levels

263 of HA, these individuals tend to be freer of worries, energetic, extroverted and optimistic. The

264 clear advantage of this pattern lies in the confidence such individuals feel when faced with danger

265 or insecurity. On the other hand, such individuals are at an increased risk of exhibiting inadequate

266 response to danger situations at the risk of actual harm. Individuals in the second group were also

267 characterized by higher levels of RD and as such were more socially interactive and sensitive.

268 They tend to be more tuned-in on social cues and to create more frequent relationships based on

269 genuine affection and concern for others. They are frequently involved in efforts to satisfy others,

270 as part of their social interactivity. These individuals were also characterized by higher levels of

271 PS and setting higher personal goals. They increase their efforts in expectation of reward and

272 view fatigue and frustration as challenges to be overcome. These individuals scored higher on

273 scales of SD, and thus tend to be more responsible, reliable and resourceful. They are more

274 realistic in setting goals and striving towards them. They also show higher levels of empathy, 
275 patience, and compassion. All these qualities lead to higher levels of cooperation and social

276 support. Individuals in this group showed lower levels of alexithymia, and a population

277 prevalence of type $\mathrm{D}$, leading to a more optimistic outlook and to less social withdrawal and

278 negative feelings.

279 Our results indicate that the personality clusters identified did not differ regarding demographic 280 characteristics including age, gender, marital status levels of education and employment. There

281 was also no difference between the groups regarding the proportion of individuals who were

282 FMS-criteria positive versus the sub-threshold individuals. Levels of widespread pain, as

283 measured by the WPI index also did not differ between groups, indicating no difference in levels

284 of chronic pain or pain distribution. The groups did however show a significant difference on the

285 symptom severity score (SSS) which was significantly higher in the first group. Thus, individuals

286 in the first group experienced higher levels of symptoms such as abdominal pain, non-refreshing

287 sleep and cognitive difficulties.

288 The results of the current study have important potential therapeutic and research implications.

289 Emotional profiles such as those identified in our study have previously been shown to be

290 associated with various aspects of wellbeing and to be predictive of an individual's future health

291 condition (Josefsson et al. 2011). Thus SD is strongly correlated to general mental health and

292 absence of personality disorder (Suchankova et al. 2011). Higher levels of SD and CO have been

293 found to be associated with better mental and physical health, increased social support and

294 improved coping with stressful situations (Zohar et al. 2011).

295 High levels of HA on the other hand have been associated with the behavioral pattern of fear and

296 avoidance (Conrad et al. 2007) and are associated with a poor response to treatment in the context

297 of chronic pain (Asmundson et al. 1999).

298 The group clustering described in the current study has the potential of leading to development of 299 patient - specific treatment plans, adjusted for the needs of FMS patients, in accordance with the 
300 style of coping identified. Thus, patients exhibiting high levels of alexithymia, may benefit from

301 interventions aimed at improving the ability to identify and express emotions, while patients

302 exhibiting characteristics of the type D personality may gain through interventions aimed at

303 improving social skills and reducing negative feelings. Targeted interventions aimed at

304 strengthening personality characteristics which boost resilience, such as identified in our second

305 group, may help patients not originally exhibiting these characteristics. Both cognitive treatment

306 and anti-depressants have previously been shown to increase levels of SD, reduce vulnerability to

307 depression and associated co-morbidities (Bulik et al. 1998; Joyce et al. 1994). Empirical testing

308 of interventions aimed at increasing SD and decreasing HA among FMS patients is a future

309 challenge.

310 Limitations:

311 While the current study appears to have identified two distinct personality patterns among

312 patients suffering from FMS and CFS, these patterns may in fact not represent a dichotomous

313 distinction, but rather a spectrum between which patients may be distributed. In addition, in the

314 present study we have not looked at the effects of modifying factors such as anxiety and

315 depression, which may well add important clinical information beyond the personality

316 characteristics. Furthermore, in the current study we have not differentiated between patients

317 suffering from FMS and those suffering from "pure" CFS (not fulfilling FMS criteria). Despite

318 the large clinical overlap between these groups, they are not identical and future research may

319 highlight

320 personality-pattern differences between these two groups.

321 Implications and conclusion:

322 In the current study we have identified two distinctive clusters of personality characteristics

323 among patients previously diagnosed as suffering from FMS or CFS. These results draw attention

324 to the heterogenic psychological characteristics of this patient population and to the necessity to 
325 avoid unwarranted generalization in characterizing these individuals. Additional research into the 326 spectrum of personality characteristics of patients suffering from FMS/CFS, as well as further

327 delineation of the differences between these groups, are called for in order to facilitate ideal 328 personalized treatment of these individuals. 
330

331 332

333 334 335

336 337

\section{$\underline{\text { References: }}$}

Afari N, and Buchwald D. 2003. Chronic fatigue syndrome: a review. American Journal of Psychiatry.

Anderberg UM, Forsgren T, Ekselius L, Marteinsdottir I, and Hallman J. 1999. Personality traits on the basis of the Temperament and Character Inventory in female fibromyalgia syndrome patients. Nordic journal of psychiatry 53:353-359.

Asmundson GJ, Norton PJ, and Norton GR. 1999. Beyond pain: the role of fear and avoidance in chronicity. Clinical psychology review 19:97-119.

Bagby RM, Parker JD, and Taylor GJ. 1994. The twenty-item Toronto Alexithymia Scale-I. Item selection and cross-validation of the factor structure. Journal of psychosomatic research 38:23-32.

Bryson GL, Turgeon AF, and Choi PT. 2012. The science of opinion: survey methods in research. Canadian Journal of Anesthesia/Journal canadien d'anesthésie 59:736-742.

Bulik CM, Sullivan PF, Joyce PR, Carter FA, and McIntosh VV. 1998. Predictors of 1-year treatment outcome in bulimia nervosa. Comprehensive Psychiatry 39:206-214.

Caprara GV, Alessandri G, Eisenberg N, Kupfer A, Steca P, Caprara MG, Yamaguchi S, Fukuzawa A, and Abela J. 2012. The positivity scale. Psychological Assessment 24:701.

Castelli L, Tesio V, Colonna F, Molinaro S, Leombruni P, Bruzzone M, Fusaro E, Sarzi-Puttini P, and Torta R. 2012. Alexithymia and psychological distress in fibromyalgia: prevalence and relation with quality of life. Clin Exp Rheumatol 30:70-77.

Celikel FC, and Saatcioglu O. 2006. Alexithymia and anxiety in female chronic pain patients. Ann Gen Psychiatry 5:13.

Clauw DJ. 2015. Fibromyalgia and related conditions. Mayo Clinic Proceedings: Elsevier. p 680692. 
354 Cloninger CR. 1986. A unified biosocial theory of personality and its role in the development of

355 anxiety states. Psychiatric developments 3:167-226.

356 Cloninger CR. 2008. The psychobiological theory of temperament and character: comment on

$357 \quad$ Farmer and Goldberg (2008).

358 Cloninger CR, Przybeck TR, and Svrakic DM. 1994. The Temperament and Character Inventory

359 (TCI): A guide to its development and use: Center for Psychobiology of Personality,

360 Washington University St. Louis, MO.

361 Cloninger CR, and Svrakic DM. 1997. Integrative psychobiological approach to psychiatric

362 assessment and treatment. Psychiatry 60:120-141.

363 Cloninger CR, Svrakic DM, and Przybeck TR. 1993. A psychobiological model of temperament

364 and character. Archives of general psychiatry 50:975-990.

365 Cloninger CR, Svrakic N, and Svrakic DM. 1997. Role of personality self-organization in

366 development of mental order and disorder. Development and psychopathology 9:881-906.

367 Cloninger CR, and Zohar AH. 2011. Personality and the perception of health and happiness.

$368 \quad$ Journal of affective disorders 128:24-32.

369 Cobb S. 1976. Social support as a moderator of life stress. Psychosomatic medicine 38:300-314.

370 Cohen H, Buskila D, Neumann L, and Ebstein RP. 2002. Confirmation of an association between

371 fibromyalgia and serotonin transporter promoter region (5-HTTLPR) polymorphism, and

372 relationship to anxiety-related personality traits. Arthritis \& Rheumatism 46:845-847.

373 Condén E, Ekselius L, and Åslund C. 2013a. Type D personality is associated with sleep

374 problems in adolescents. Results from a population-based cohort study of Swedish

375 adolescents. Journal of psychosomatic research 74:290-295.

376 Condén E, Leppert J, Ekselius L, and Åslund C. 2013b. Type D personality is a risk factor for psychosomatic symptoms and musculoskeletal pain among adolescents: a cross-sectional study of a large population-based cohort of Swedish adolescents. BMC pediatrics 13:1. 
379 Conrad R, Schilling G, Bausch C, Nadstawek J, Wartenberg HC, Wegener I, Geiser F,

380 Imbierowicz K, and Liedtke R. 2007. Temperament and character personality profiles and

381 personality disorders in chronic pain patients. PAIN® 133:197-209.

382 Conrad R, Wegener I, Geiser F, and Kleiman A. 2013. Temperament, character, and personality

383 disorders in chronic pain. Current pain and headache reports 17:1-9.

384 Dan B, Hagit C, Lily N, and Ebstein R. 2004. An association between fibromyalgia and the

385 dopamine D4 receptor exon III repeat polymorphism and relationship to novelty seeking

$386 \quad$ personality traits. Molecular psychiatry 9:730-731.

387 Denollet J. 2005. DS14: standard assessment of negative affectivity, social inhibition, and Type D

388 personality. Psychosomatic medicine 67:89-97.

389 Denollet J, Rombouts H, Gillebert T, Brutsaert D, Sys S, and Stroobant N. 1996. Personality as

390 independent predictor of long-term mortality in patients with coronary heart disease. The

$391 \quad$ Lancet 347:417-421.

392 Gencay-Can A, and Can SS. 2012. Temperament and character profile of patients with

393 fibromyalgia. Rheumatology international 32:3957-3961.

394 Giesecke T, Williams DA, Harris RE, Cupps TR, Tian X, Tian TX, Gracely RH, and Clauw DJ.

395 2003. Subgrouping of fibromyalgia patients on the basis of pressure-pain thresholds and

396 psychological factors. Arthritis \& Rheumatism 48:2916-2922.

397 Glazer Y, Buskila D, Cohen H, Ebstein R, and Neumann L. 2010. Differences in the personality

398 profile of fibromyalgia patients and their relatives with and without fibromyalgia.

$399 \quad$ Clinical and Experimental Rheumatology-Incl Supplements 28:S27.

400 Heath AC, Cloninger CR, and Martin N. 1994. Testing a model for the genetic structure of

401 personality: a comparison of the personality systems of Cloninger and Eysenck. Journal

402 of personality and social psychology 66:762. 
403 Josefsson K, Cloninger CR, Hintsanen M, Jokela M, Pulkki-Råback L, and Keltikangas-Järvinen

404 L. 2011. Associations of personality profiles with various aspects of well-being: a

405 population-based study. Journal of affective disorders 133:265-273.

406 Joyce PR, Mulder RT, and Cloninger CR. 1994. Temperament predicts clomipramine and

407 desipramine response in major depression. Journal of affective disorders 30:35-46.

408 Kozma A, Stone S, and Stones M. 2000. Stability in components and predictors of subjective

409 well-being (SWB): Implications for SWB structure. Advances in quality of life theory

$410 \quad$ and research: Springer, 13-30.

411 Leombruni P, Zizzi F, Miniotti M, Colonna F, Castelli L, Fusaro E, and Torta R. 2016. Harm

412 Avoidance and Self-Directedness characterize fibromyalgic patients and the symptom

413 severity. Frontiers in Psychology 7. 10.3389/fpsyg.2016.00579

414 Lumley MA, Smith JA, and Longo DJ. 2002. The relationship of alexithymia to pain severity and 415 impairment among patients with chronic myofascial pain: comparisons with self-efficacy, 416 catastrophizing, and depression. Journal of psychosomatic research 53:823-830.

417 Malin K, and Littlejohn GO. 2012. Personality and fibromyalgia syndrome. The open 418 rheumatology journal 6:273.

419 Passmore C, Dobbie AE, Parchman M, and Tysinger J. 2002. Guidelines for constructing a 420 survey. FAMILY MEDICINE-KANSAS CITY- 34:281-286.

421 Procidano ME, and Heller K. 1983. Measures of perceived social support from friends and from 422 family: Three validation studies. American journal of community psychology 11:1-24.

423 Sarason IG, Sarason BR, Potter III EH, and Antoni MH. 1985. Life events, social support, and $424 \quad$ illness. Psychosomatic medicine 47:156-163.

425 Sayar K, Gulec H, and Topbas M. 2004. Alexithymia and anger in patients with fibromyalgia. $426 \quad$ Clinical rheumatology 23:441-448. 
427 Schoofs N, Bambini D, Ronning P, Bielak E, and Woehl J. 2004. Death of a lifestyle: the effects

428 of social support and healthcare support on the quality of life of persons with

429 fibromyalgia and/or chronic fatigue syndrome. Orthopaedic Nursing 23:364-374.

430 Steinweg DL, Dallas AP, and Rea WS. 2011. Fibromyalgia: unspeakable suffering, a prevalence $431 \quad$ study of alexithymia. Psychosomatics 52:255-262.

432 Suchankova P, Baghaei F, Rosmond R, Holm G, Anckarsäter H, and Ekman A. 2011. Genetic 433 variability within the S100B gene influences the personality trait self-directedness. 434 Psychoneuroendocrinology 36:919-923.

435 Tuzer V, Bulut SD, Bastug B, Kayalar G, Göka E, and Beştepe E. 2011. Causal attributions and 436 alexithymia in female patients with fibromyalgia or chronic low back pain. Nordic journal of psychiatry 65:138-144.

438 Wolfe F, Clauw DJ, Fitzcharles M-A, Goldenberg DL, Häuser W, Katz RS, Mease P, Russell AS, 439 Russell IJ, and Winfield JB. 2011. Fibromyalgia criteria and severity scales for clinical and epidemiological studies: a modification of the ACR Preliminary Diagnostic Criteria

Wolfe F, Clauw DJ, Fitzcharles MA, Goldenberg DL, Katz RS, Mease P, Russell AS, Russell IJ, 443 Winfield JB, and Yunus MB. 2010. The American College of Rheumatology preliminary diagnostic criteria for fibromyalgia and measurement of symptom severity. Arthritis care \& research 62:600-610.

Zimet GD, Dahlem NW, Zimet SG, and Farley GK. 1988. The multidimensional scale of perceived social support. Journal of personality assessment 52:30-41.

448 Zohar AH, and Cloninger CR. 2011. The psychometric properties of the TCI-140 in Hebrew. European Journal of Psychological Assessment. 
450 Zohar AH, Denollet J, Ari LL, and Cloninger CR. 2011. The psychometric properties of the DS14 451 in Hebrew and the prevalence of type D personality in Israeli adults. European Journal of $452 \quad$ Psychological Assessment. 